\title{
Mediating ERK1/2 signaling rescues congenital heart defects in a mouse model of Noonan syndrome
}

\author{
Tomoki Nakamura, ${ }^{1}$ Melissa Colbert, ${ }^{1}$ Maike Krenz,, Jeffery D. Molkentin, ${ }^{1}$ Harvey S. Hahn, ${ }^{2}$ \\ Gerald W. Dorn II, ${ }^{2}$ and Jeffrey Robbins ${ }^{1}$ \\ ${ }^{1}$ Cincinnati Children's Hospital Medical Center, The Children's Hospital Research Foundation, Division of Molecular Cardiovascular Biology, Cincinnati, \\ Ohio, USA. ${ }^{2}$ Department of Internal Medicine and Department of Cardiology, University of Cincinnati Medical Center, Cincinnati, Ohio, USA.
}

\begin{abstract}
Noonan syndrome (NS) is an autosomal dominant disorder characterized by a wide spectrum of defects, which most frequently include proportionate short stature, craniofacial anomalies, and congenital heart disease (CHD). NS is the most common nonchromosomal cause of CHD, and $80 \%-90 \%$ of NS patients have cardiac involvement. Mutations within the protein tyrosine phosphatase Src homology region 2, phosphatase 2 (SHP2) are responsible for approximately $50 \%$ of the cases of NS with cardiac involvement. To understand the developmental stage- and cell type-specific consequences of the NS SHP2 gain-of-function mutation, Q79R, we generated transgenic mice in which the mutated protein was expressed during gestation or following birth in cardiomyocytes. Q79R SHP2 embryonic hearts showed altered cardiomyocyte cell cycling, ventricular noncompaction, and ventricular septal defects, while, in the postnatal cardiomyocyte, Q79R SHP2 expression was completely benign. Fetal expression of Q79R led to the specific activation of the ERK1/2 pathway, and breeding of the Q79R transgenics into ERK1/2-null backgrounds confirmed the pathway's necessity and sufficiency in mediating mutant SHP2's effects. Our data establish the developmental stage-specific effects of Q79R cardiac expression in NS; show that ablation of subsequent ERK1/2 activation prevents the development of cardiac abnormalities; and suggest that ERK1/2 modulation could have important implications for developing therapeutic strategies in CHD.
\end{abstract}

\section{Introduction}

Noonan syndrome (NS) is an autosomal dominant disorder characterized by a wide spectrum of defects, which most frequently include proportionate short stature, craniofacial anomalies, and congenital heart disease (CHD) (1-3). Mild mental retardation and hematological disorders such as bleeding diathesis and juvenile myelomonocytic leukemia are also occasionally observed in affected individuals (3-5). NS occurs with an estimated incidence of 1 in 1,000-2,500 live births (1) and is the most common nonchromosomal cause of CHD. Among NS patients, $80 \%-90 \%$ have cardiac involvement $(2,6)$, with pulmonic stenosis commonly presenting, while atrioventricular septal defects and hypertrophic cardiomyopathy (HCM) occur at a lower frequency (7).

Germline missense mutations of human PTPN11, which encodes SHP2 (Src homology region 2, phosphatase 2), occur in approximately $50 \%$ of NS patients (8). SHP2 is ubiquitously expressed and is required during embryonic development $(9,10)$. SHP2 is involved in signaling pathways controlling morphogenic movements during gastrulation (9), limb development (11), valvulogenesis (12), and hematopoietic cell progenitor commitment and differentiation $(13,14)$. The protein has $2 \mathrm{SH} 2$ domains $(\mathrm{N}-\mathrm{SH} 2$ and $\mathrm{C}-\mathrm{SH} 2$ ) and a protein tyrosine phosphatase (PTP) domain near the carboxyl terminus, with $\mathrm{N}-\mathrm{SH} 2$ functioning as an intra-

Nonstandard abbreviations used: CHD, congenital heart disease; HCM, hypertrophic cardiomyopathy; MHC, myosin heavy chain; NF1, neurofibromatosis type 1; NS, Noonan syndrome; Ntg, nontransgenic; PTP, protein tyrosine phosphatase; SHP2, Src homology region 2, phosphatase 2; VSD, ventricular septal defect.

Conflict of interest: The authors have declared that no conflict of interest exists. Citation for this article: J. Clin. Invest. 117:2123-2132 (2007). doi:10.1172/JCI30756 molecular switch to control SHP2's catalytic activity (15). Earlier studies showed that SHP2 mutations in NS mostly cluster in the $\mathrm{N}-\mathrm{SH} 2$ and PTP functional domains. Activated mutants of SHP2 were described in Xenopus (16), and subsequently, energetics-based structural analyses showed that some mutations stabilize SHP2 in the active conformation, leading to a gain of function $(8,17)$. A knock-in mouse model with the NS-causative D61G mutation exhibited cardiac defects, some of which were similar to human disease presentation (18), supporting a causal role for SHP2's gain-of-function mutation in cardiac pathogenesis, including valve abnormalities. Recently, we (19) and others (20) reported the effects of an NS SHP2 mutation on cushion cells and cardiomyocytes, respectively. However, the molecular, cellular, and developmental influences of SHP2 mutations on both the developing and mature heart remain unclear.

In the mouse, Ptpn11 is expressed ubiquitously early in development, including in the developing (and mature) heart. Here we explore the developmental stage-specific mechanisms of the NS SHP2 mutation Q79R in the cardiomyocyte population. Fetal ventricular expression of Q79R selectively activated the ERK1/2 pathway, resulting in aberrant cardiac architecture, while expression of the mutation after birth in cardiomyocytes was benign. During cardiac development, cardiomyocyte ERK1/2 activation appears to be sufficient to cause the cardiac defects, as ERK1/2 ablation during embryogenesis rescued the mutant phenotype and restored normal cardiac anatomy and function.

\section{Results}

Expression of Q79R in the cardiomyocytes leads to different outcomes depending upon the developmental time. To investigate the gain-of-function 


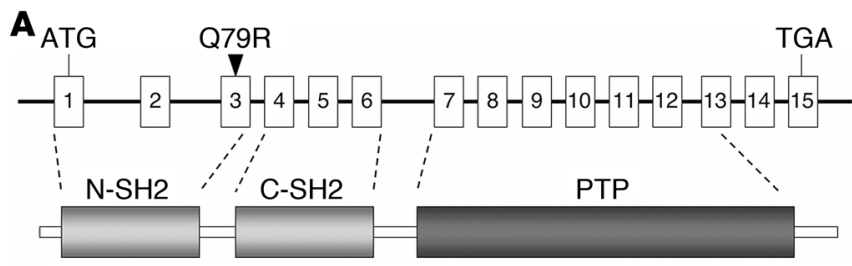

B Mouse cardiac $\beta-\mathrm{MHC}$ promoter $\square$

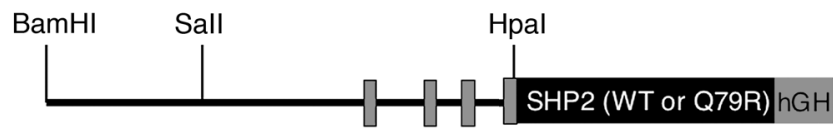
$\lceil$ Mouse cardiac $\alpha-\mathrm{MHC}$ promoter $\square$

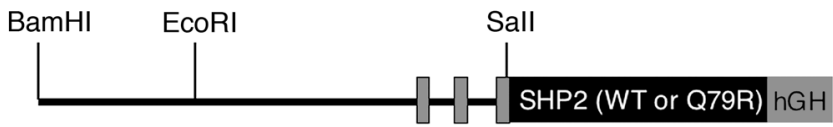

C

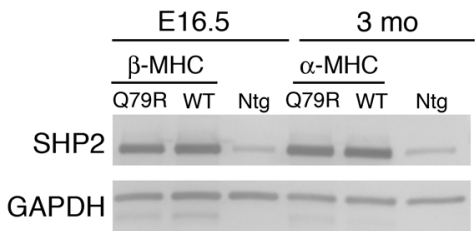

D

$\frac{\mathrm{E} 9.5}{\alpha-\mathrm{MHC} C} \frac{\mathrm{E} 11.5}{\alpha-\mathrm{MHC}} \frac{\mathrm{E} 13.5}{\alpha-\mathrm{MHC}} \frac{\mathrm{E} 16.5}{\alpha-\mathrm{MHC}}$

$\mathrm{SHP2}$

Ni Q79R Nig Q79R Ntg Q79R Ntg Q79R

GAPDH

$-$

effect of the NS SHP2 mutation on the heart, we generated Tg mice in which normal, WT and mutated SHP2 was expressed specifically in cardiomyocytes, either during gestation or after birth. The most frequently found mutation in the N-SH2 domain of SHP2, Q79R (17), which has a 4.5-fold higher phosphatase activity than that of WT SHP2 (19), was used for this study. The $\beta$ - and $\alpha$-myosin heavy chain ( $\beta$-MHC and $\alpha$-MHC) promoters were used to express WT and Q79R SHP2 in the fetal and postnatal ventricles, respectively (Figure 1, A and B). Multiple lines were established (Supplemental Figure 1; supplemental material available online with this article; doi:10.1172/JCI30756DS1), and, after expression levels were determined for each Tg construct, we selected single lines of mice showing roughly equivalent increases in cardiac SHP2 protein content for subsequent analyses (Figure 1C). To confirm the developmental segregation of the promoters - that is, inactivity of the $\alpha$-MHC promoter during ventricular development - total SHP2 protein was measured from E9.5 through E16.5 in the $\alpha$-MHC Q79R line shown in Figure 1C. As expected, SHP2 protein levels did not differ between the nontransgenic (Ntg) and Tg animals (Figure 1D).

At E16.5, ventricular noncompaction, ventricular septal defects (VSDs), and abnormal anatomy of the interventricular groove were prominent in the $\beta$-MHC Q79R hearts, a phenotype similar to that reported in another mouse model of NS, in which the D61G SHP2 mutation was expressed (18). When multiple lines showing varying degrees of overexpression were analyzed, the severity and frequency of the defects were both dose dependent (Supplemental Figure 1). In contrast, $\alpha$-MHC WT, $\alpha$-MHC Q79R, and $\beta$-MHC WT hearts showed no aberrant ventricular architecture (Figure 2A). Cardiac

\section{Figure 1}

Developmental stage-specific transgene expression of normal or mutant SHP2 in the ventricles. (A) Schematic diagram of Ptpn11 showing the approximate exon boundaries for the functional domains and location of the Q79R mutation. (B) Constructs used to generate cardiomyocyte-specific expression of normal (WT) or NS mutant Q79R protein. Mouse cDNA containing either normal (WT) or mutant (Q79R) SHP2 was linked to either the $\beta$ - or $\alpha$-MHC promoter to express the transgene. The $\beta-\mathrm{MHC}$ promoter expresses the transgene in the ventricular cardiomyocytes between E9.25 and E19, while the $\alpha-\mathrm{MHC}$ promoter expresses the transgene in the postnatal ventricle. Vertical boxes in $\beta-\mathrm{MHC}$ and $\alpha-\mathrm{MHC}$ promoters represent noncoding exons in $5^{\prime}$-untranslated region. A poly adenylation signal sequence derived from the human growth hormone gene (hGH) was inserted downstream of the cDNA. (C) Western blot analyses of Tg cardiac tissue. In $\beta$-MHC hearts, SHP2 protein expression was increased 5.3-fold in both the WT and Q79R Tg hearts at E16.5. SHP2 protein expression was increased 11.4- and 13.6-fold in the $\alpha-M H C$ WT and Q79R hearts, respectively, at 3 months after birth. (D) To confirm that the $\alpha-M H C-$ driven SHP2 construct was transcriptionally inactive during gestation, total SHP2 protein in the Q79R line (13.6-fold overexpression after birth) was measured at E9.5-E16.5, the period during which compaction occurs. When values were normalized to a GAPDH loading control, no statistically significant increases in protein levels were observed.

histology at 3 months showed that significant SHP2 overexpression in the postnatal cardiomyocytes had no effects in either the $\alpha$-MHC WT or Q79R hearts. In the $\beta$-MHC Q79R hearts, embryonic ventricular noncompaction persisted postnatally (Figure $2 \mathrm{~B}$ ), with no evidence of fibrosis (Figure 2C).

We further analyzed the effect of SHP2 expression on cardiac function and fate in these mice. Echocardiography revealed that $\beta$-MHC Q79R hearts showed markedly decreased contractility and ventricular wall thickness, while cardiac function was normal in $\alpha$-MHC WT, $\alpha$-MHC Q79R, and $\beta$-MHC WT mice (Table 1). Cardiac hypofunction in $\beta$-MHC Q79R mice resulted in congestive heart failure (Figure 2D). Animals typically presented postmortem with atrial and ventricular dilatation, chronic left atrial thrombi, and abnormal anatomy of the intraventricular groove. Heart weight to body weight (HW/BW) ratios and lung weight to body weight (LW/BW) ratios were each increased (Figure 2, E and F). In $\beta$-MHC Q79R mice, 38.8\% (19 of 49) died by 8 months, while all Ntg $(n=32), \alpha-\mathrm{MHC}$ WT $(n=37), \alpha-\mathrm{MHC}$ Q79R $(n=50)$, and $\beta$-MHC WT $(n=32)$ mice survived.

Q79R SHP2 expression selectively activates ERK1/2. SHP2 regulates intracellular signaling pathways that help control cell proliferation, differentiation, migration, adhesion, and survival (15) by modulating MAPK cascades (21-24). We thus examined several candidate downstream signaling pathways for activation by the Q79R SHP2 mutation during gestation. Consistent with previous in vitro data (19), the ERK1/2 branch was hyperphosphorylated in $\beta$-MHC Q79R hearts (Figure 3A and Supplemental Table 1) but not in $\alpha$-MHC Q79R hearts (data not shown) during gestation, consistent 

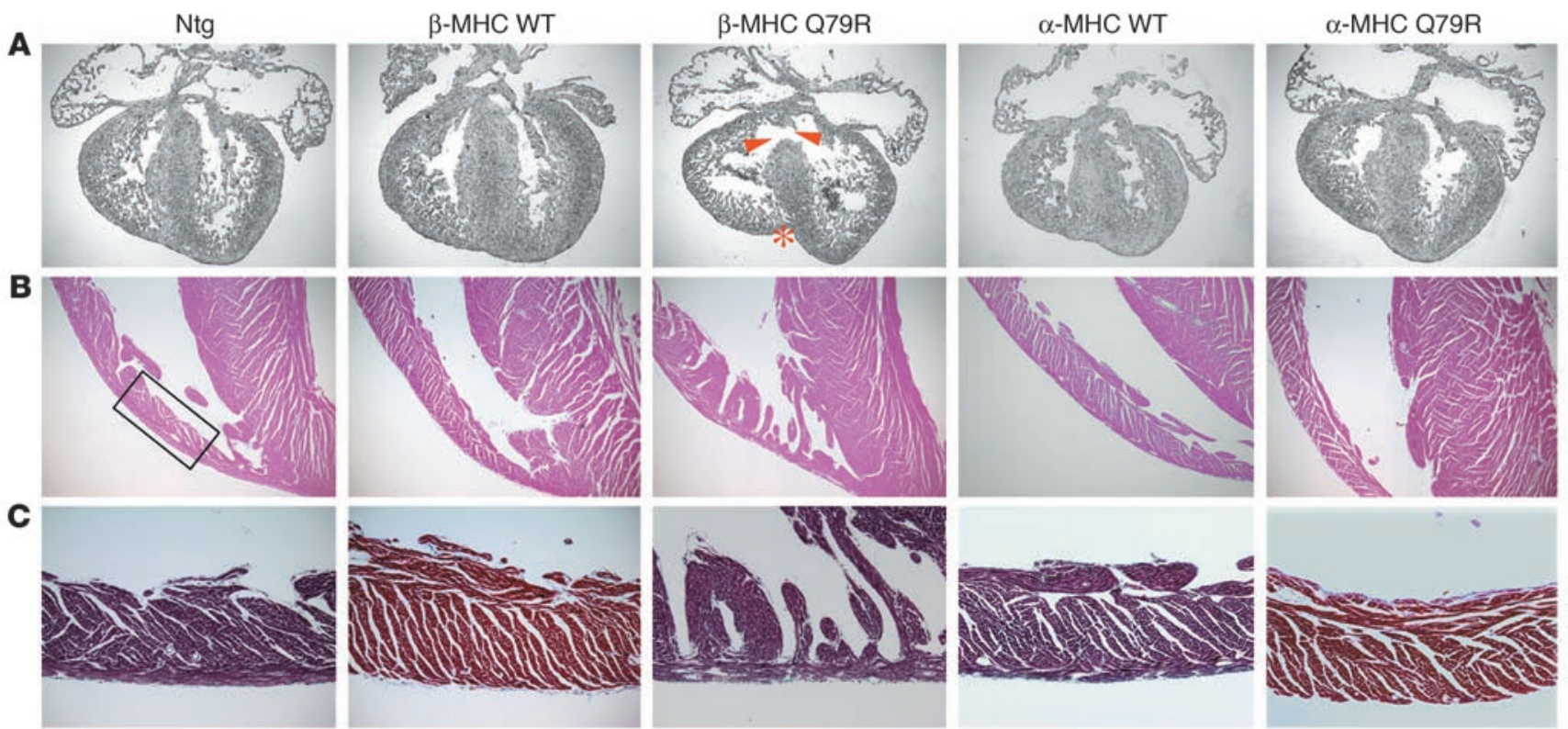

D
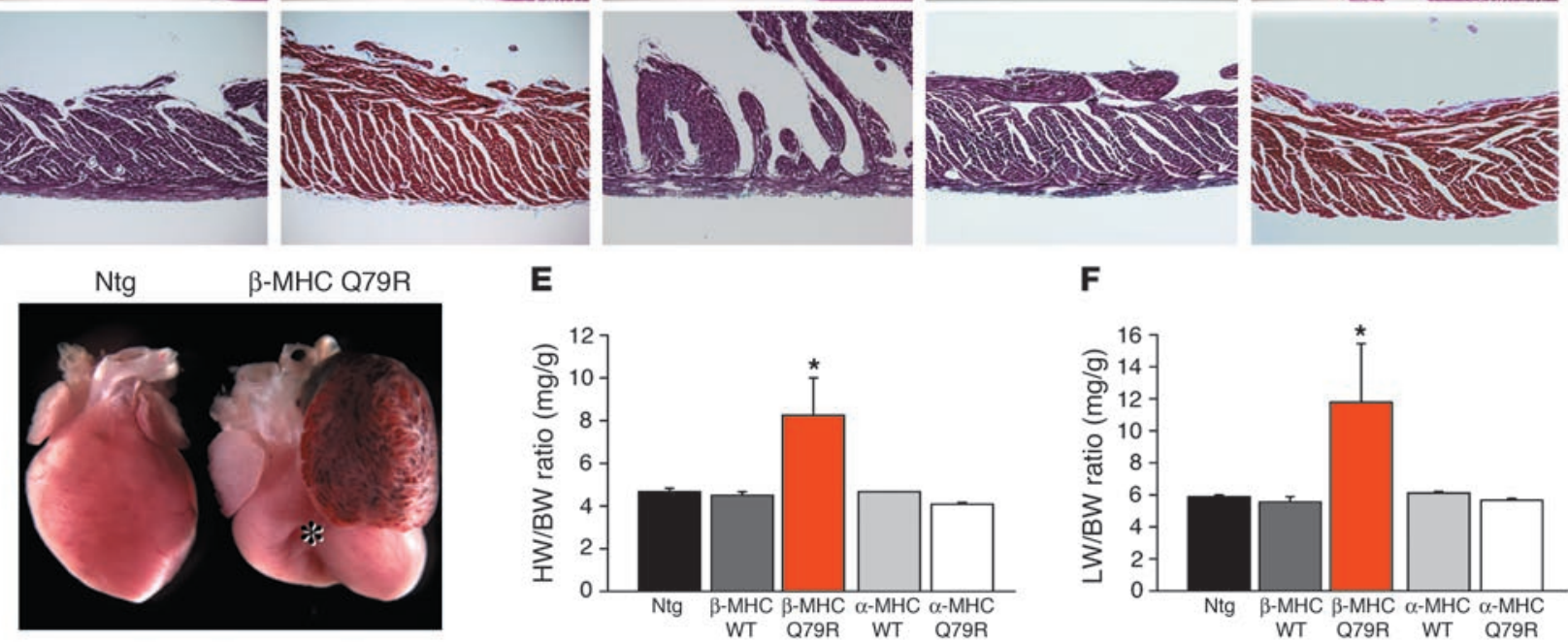

$\mathbf{F}$

\section{Figure 2}

Histological findings. (A) Four-chamber view of the hearts at E16.5 (original magnification, $\times 4$ ). Ventricular noncompaction, VSDs (arrowheads), and abnormal anatomy of the interventricular groove $\left(^{*}\right)$ are prominent in the $\beta-\mathrm{MHC}$ Q79R hearts, while the $\alpha-\mathrm{MHC}$ WT and Q79R and $\beta$-MHC WT hearts appear to be normal. (B) Longitudinal ventricular sections at 3 months after birth, stained with H\&E (original magnification, $\times 4$ ). Each image shows the right-ventricular free wall on the left and the ventricular septum on the right. Despite SHP2 overexpression in the postnatal cardiomyocytes, no abnormalities were seen in the $\alpha-\mathrm{MHC}$ WT and Q79R hearts. In the $\beta-\mathrm{MHC}$ Q79R hearts, the ventricular noncompaction observed in the embryonic stage continued to present postnatally. (C) Masson trichrome staining of the right-ventricular free walls from the rectangular area indicated in $\mathbf{B}$ (original magnification, $\times 10$ ). For each panel at least 5 hearts were analyzed. For all panels, orientation is apical on the right and basal on the left. (D) Representative photograph of an end-stage failing heart in the $\beta$-MHC Q79R mouse (6 weeks old; original magnification, $\times 2.8$ ). Compared with those of the Ntg littermates, $\beta$-MHC Q79R hearts showed both atrial and ventricular dilatation and abnormal interventricular groove anatomy $\left(^{*}\right)$. Left-ventricular hypofunction caused regurgitation toward the left atrium, resulting in massive thrombi. (E and $\mathbf{F}$ ) Analyses of heart weight to body weight (HW/BW) ratio and lung weight to body weight (LW/BW) ratio in age- and sex-matched mice (3 months after birth, $n=8$ [4 male, 4 female]). HW/BW and LW/BW ratios were increased in $\beta$-MHC Q79R mice. ${ }^{\star} P<0.005$.

with the activities of the 2 promoters during cardiac development (25). ERK1/2 hyperactivation was sustained throughout the latter half of gestation in the $\beta$-MHC Q79R hearts (Figure 3, B-D). We further examined ERK1/2 in the adult $\beta$-MHC Q79R hearts and asked whether expression varied in accordance with cardiac dysfunction. Interestingly, ERK1/2 activation was downregulated postnatally even in the failing hearts (Figure 3B, right). ERK1/2 was hyperphosphorylated in the $\alpha$-MHC Q79R hearts at 3 months (Figure $3 \mathrm{~A}$ ), suggesting that the signaling activity parallels SHP2 expression, but it appears that chronic activation of this pathway in cardiomyocytes after cardiac organogenesis is largely benign, as these mice had normal lifespans, conserved cardiac function (Table 1), and no pathology (Figure 2). The other MAPK cascades, including p38, JNK1/2, and ERK5, were not activated (Figure 3A and Supplemental Table 1). It has been reported that SHP2 can also participate in cell survival, migration, adhesion, and myogenesis by signaling through the Akt, JAK/STAT, or RhoA pathways (26-29). However, the expression of these proteins or the respective effectors (Akt, STAT1, $-3,-5$, and MYPT) were all unchanged in the cardiomyocyte population (Figure 3A and Supplemental Table 1).

Reduction of ERK1/2 byperphosphorylation in $\beta-M H C Q 79 R$ hearts. Both ERK1 and ERK2 are widely expressed throughout development (30). ERK1/2 activation is required for embryogenesis (31), with the duration and magnitude of ERK activity differing among cell types (32). In the mouse, brief pulses of ERK1/2 activation occur in the heart primordia, suggesting that ERK1/2 signal- 
Table 1

Cardiac function in $\alpha$ - and $\beta$-MHC SHP2 mice

$\begin{array}{lccccc} & & & & & \\ & \text { Ntg } & \beta-\text { MHC WT } & \beta-\text { MHC Q79R } & \alpha-\text { MHC WT } & \alpha \text {-MHC Q79R } \\ \text { Heart rate (bpm) } & 713 \pm 14 & 697 \pm 11 & 698 \pm 8 & 691 \pm 15 & 713 \pm 15 \\ \text { IVST (mm) } & 0.74 \pm 0.01 & 0.73 \pm 0.04 & 0.55 \pm 0.03^{\mathrm{A}, \mathrm{B}, \mathrm{C}} & 0.71 \pm 0.04 & 0.66 \pm 0.02 \\ \text { LVPWT (mm) } & 0.76 \pm 0.02 & 0.76 \pm 0.05 & 0.59 \pm 0.03^{\mathrm{A}, \mathrm{C}} & 0.67 \pm 0.02 & 0.65 \pm 0.02 \\ \text { LVDd (mm) } & 3.22 \pm 0.08 & 3.30 \pm 0.07 & 3.62 \pm 0.12^{\mathrm{B}} & 3.22 \pm 0.12 & 3.28 \pm 0.08 \\ \text { LVDs (mm) } & 1.72 \pm 0.08 & 1.87 \pm 0.06 & 2.82 \pm 0.14 \mathrm{~A}, \mathrm{~B}, \mathrm{C}, \mathrm{D} & 1.70 \pm 0.09 & 1.83 \pm 0.08 \\ \text { FS (\%) } & 46.64 \pm 1.63 & 44.35 \pm 1.35 & 22.22 \pm 1.84^{\mathrm{A}, \mathrm{B}, \mathrm{C}, \mathrm{D}} & 47.50 \pm 1.25 & 44.30 \pm 1.91 \\ \text { Vcfc } & 10.80 \pm 0.49 & 8.32 \pm 0.65 & 4.41 \pm 0.83^{\mathrm{A}, \mathrm{B}, \mathrm{C}, \mathrm{D}} & 10.34 \pm 0.45 & 9.69 \pm 0.55 \\ & & & & \end{array}$

Age- and sex-matched mice ( 15 weeks after birth, $n=8$ [ 4 male, 4 female]) were assessed by M-mode echocardiography. FS, fractional shortening; IVST, interventricular wall thickness; LVDd, left-ventricular end-diastolic dimension; LVDs, left-ventricular end-systolic dimension; LVPWT, left-ventricular posterior wall thickness; Vcfc, heart rate-corrected velocity of circumferential shortening. Data are expressed as mean \pm SEM. One-way ANOVA was used for parametric comparisons, and significance of individual differences was evaluated using Scheffé test if ANOVA was significant. ${ }^{A} P<0.05$ compared with $\mathrm{Ntg} .{ }^{B} P<0.05$ compared with $\alpha$-MHC WT. ${ }^{C} P<0.05$ compared with $\beta$-MHC WT. DP $<0.05$ compared with $\alpha$-MHC Q79R.

mice to Erk1- and Erk2-knockout mice, respectively. Homozygous Erk1-null (Erk1 $\left.{ }^{-/}\right)$mice are viable and fertile, although thymocyte proliferation and maturation are affected (33). Homozygous loss of Erk2 (Erk2 $\left.2^{--}\right)$results in embryonic lethality, but the heterozygotes survive into adulthood (30). Thus, we generated $\beta$-MHC Q79R $\times$ Erk1 $^{-/-}$ and $\beta$-MHC Q79R $\times$ Erk2 $^{+/-}$mice. At E16.5, ERK1 hyperactivation was completely ablated in the $\beta$-MHC Q79R $\times$ Erk1 $^{-1-}$ hearts, and ERK2 hyperactivation was substantially reduced in the MHC Q79R $\times$ Erk2 $^{+/-}$hearts (Figure 4, A and B). Similar results were observed at E11.5 and E13.5 as well (data not

ing plays a transient and dynamic role in cardiac development, although the specific role of each ERK isoform remains unclear (31). Studies with ERK1- and ERK2-knockout mice showed that ablation of one ERK did not influence the expression of the other ERK isoform, suggesting at least some degree of autonomy (30, 33). We hypothesized that sustained cardiomyocyte ERK1/2 hyperphosphorylation during embryogenesis had specific adverse effects on cardiac maturation, leading to ventricular noncompaction and VSDs. To establish the sufficiency of ERK1/2 hyperphosphorylation's effects on cardiac development, we crossed the $\beta$-MHC Q79R shown). In both $\beta$-MHC Q79R $\times$ Erk1 $^{-1-}$ and $\beta$-MHC Q79R $\times$ Erk2 $^{+/-}$fetal hearts (E16.5), the ventricular noncompaction and concomitant thinning of the compact myocardium found in the $\beta$-MHC Q79R hearts were significantly decreased (Figure 4C). These changes are more readily apparent at higher magnification (Figure 4D). Qualitative observations were confirmed by quantitation (Figure 4E), and the frequency of VSDs at E16.5 was also decreased: Ntg $(n=10), 0$ VSDs; Erk1 $1^{-/-}(n=9), 1 \mathrm{VSD} ;$ Erk2 $^{+/-}$ $(n=10)$, 0 VSDs; $\beta$-MHC Q79R $(n=10), 7$ VSDs; $\beta$-MHC Q79R $\times \operatorname{Erk1}^{-/-}(n=11), 2$ VSDs; $\beta$-MHC Q79R $\times \operatorname{Erk2}^{+/-}(n=10)$,

\section{Figure 3}

SHP2 Q79R expression results in ERK1/2 hyperphosphorylation. (A) Western blots of the signaling pathways potentially downstream of SHP2. ERK1/2 is hyperphosphorylated in $\beta$-MHC Q79R (E16.5) and $\alpha-M H C$ Q79R (3-month) hearts. The phosphorylated species were quantitated and the values given in Supplemental Table 1. (B) ERK1/2 is hyperphosphorylated throughout the latter half of gestation in the $\beta-\mathrm{MHC}$ Q79R hearts but this does not occur postnatally, when the $\beta$-MHC promoter is inactive. All lanes contained pooled protein from at least 5 hearts. Quantitation of the blots is shown in the Supplemental Table 1. (C and D) Quantitation of p-ERK1 and $p$-ERK2, respectively. The blots shown in B were quantitated using a Storm Phosphorlmager, as described in Methods. CHF, congestive heart failure; NF, nonfailing.
A
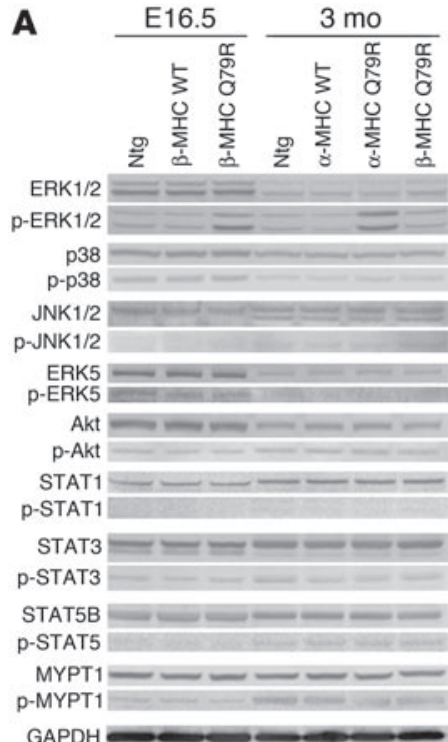

B

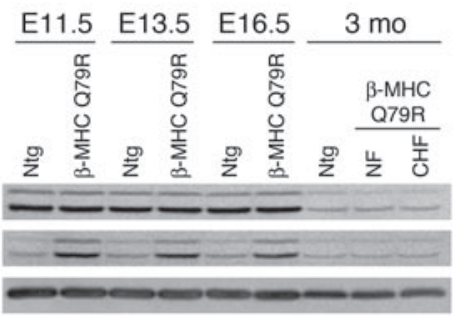

C

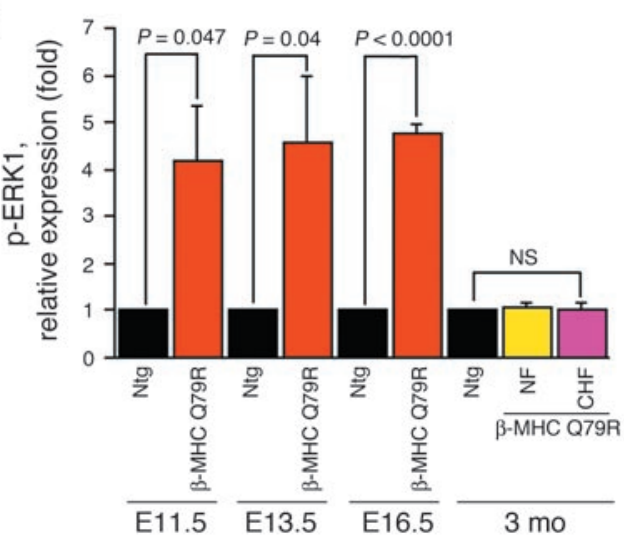

D

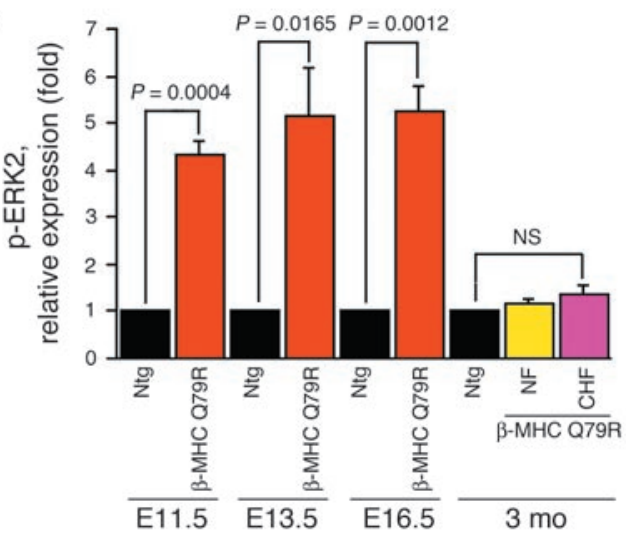




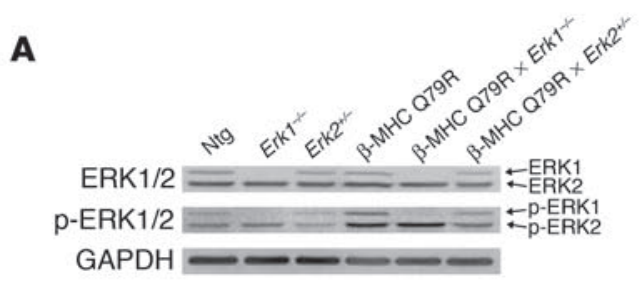

C
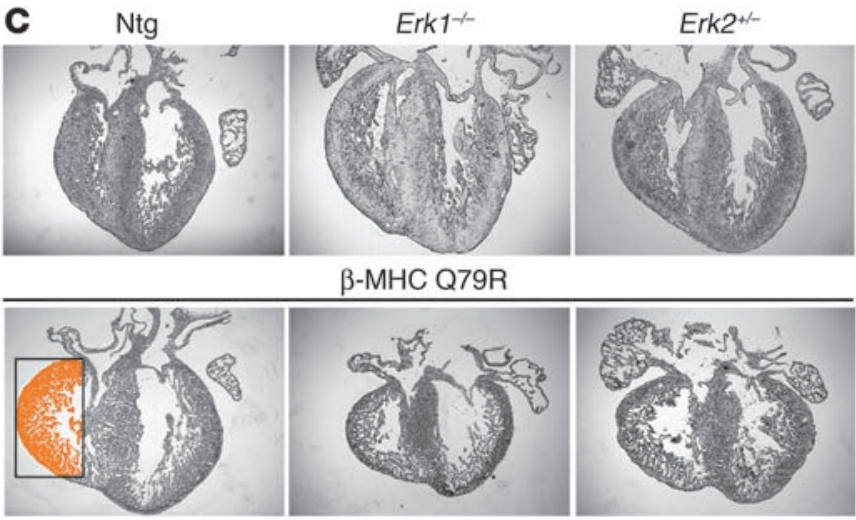

$\beta-\mathrm{MHC}$ Q79R $\times$ Erk1 $^{-1-}$

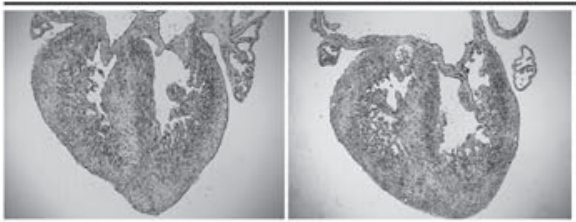

$\beta-\mathrm{MHC}$ Q79R $\times \mathrm{Erk2}^{+/-}$
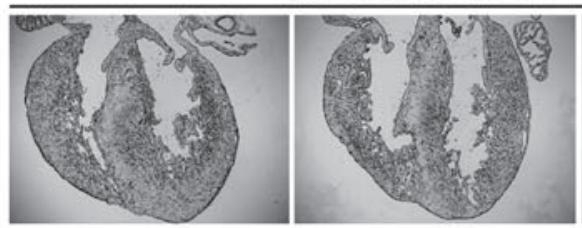

B

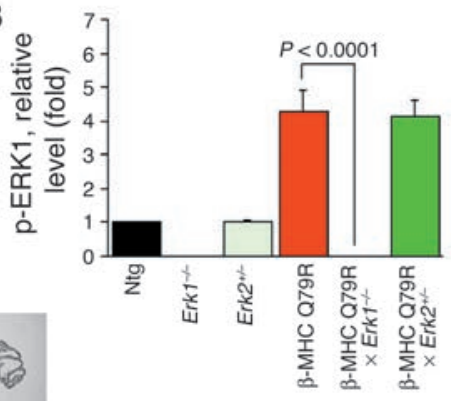

D

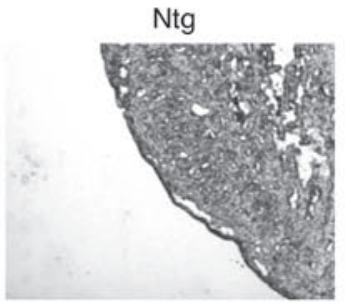

$\beta-\mathrm{MHC}$ Q79R $\times$ Erk1 ${ }^{--}$

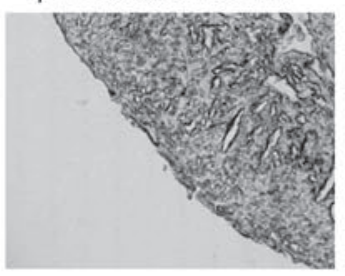

$\mathbf{E}$

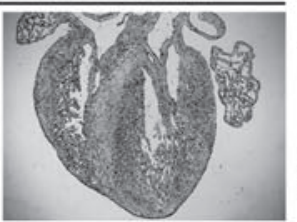

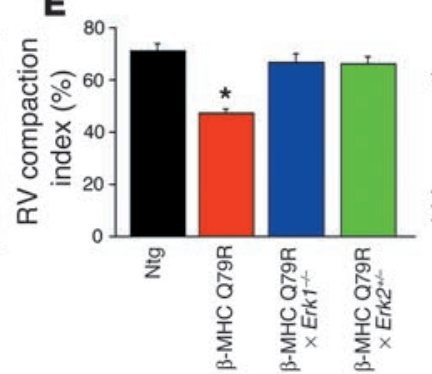

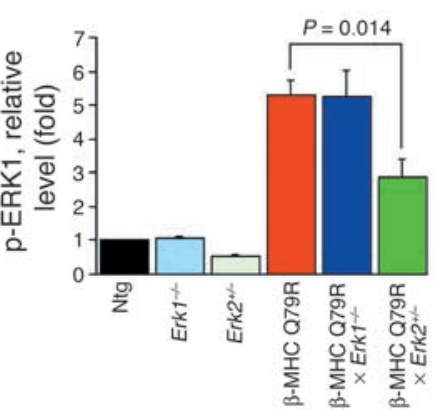

B-MHC Q79R

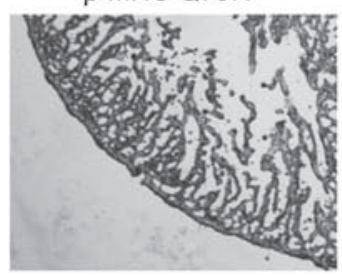

$\beta-\mathrm{MHC}$ Q79R $\times$ Erk2 $^{+/}$
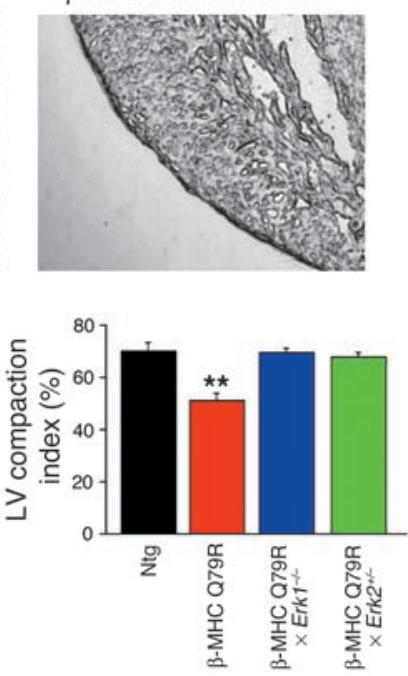

Figure 4

Reduction of ERK1/2 hyperphosphorylation rescues Q79R-induced heart disease. Western blot analysis (A) of E16.5 hearts and quantitation (B). ERK1 and ERK2 hyperphosphorylation in the $\beta$-MHC Q79R heart is reduced after crossing to the Erk $1^{-/-}$and Erk2+/- mice. (C) Histology of the E16.5 hearts shows that the ventricular noncompaction and concomitant wall thinning seen in $\beta$-MHC Q79R hearts are ameliorated when the mice are crossed to the Erk1-/- and Erk2+-- mice. (D) Higher magnifications of a subsection of the hearts shown in C. Identically placed, small areas derived from the apical side of the right-ventricular free wall were selected and clearly show the thinning present in the Q79R mutants, compared with either the WT hearts or the hearts derived from the Erk1 $1^{-/}$and Erk2 ${ }^{+-}$crosses. (E) Quantitation of ventricular compaction at E16.5. The compaction index was determined using Metamorph software (version 6.3) as described in Methods. RV, right ventricle; LV, left ventricle. ${ }^{\star} P<0.0001$ in RV; ${ }^{* *} P<0.0001$ in LV $(n=7)$. Vent., ventricular.

1 VSD). The data indicate that both ERK1 and ERK2 activation are required for the full phenotype to present.

We then analyzed the histology and cardiac function of postnatal hearts derived from these crosses to see whether the rescue persisted. In $\beta$-MHC Q79R $\times$ Erk $^{-/-}$and $\beta$-MHC Q79R $\times$ Erk2 ${ }^{+/-}$mice, ventricular noncompaction was rescued in both the fetal and adult hearts (Figure 5A). Echocardiography at 15 weeks demonstrated significant improvements in both ventricular wall thickness and contractility (Table 2), with HW/BW and LW/BW ratios indistinguishable from those of $\mathrm{Ntg}$ hearts (Figure 5B). The data show that reduction of inappropriate ERK1 or ERK2 hyperphosphorylation during embryogenesis restores normal cardiac anatomy and function.

ERK not only mediates cell survival but also regulates cell differentiation and proliferation (32). We observed no changes in caspase- 3 activity or in the levels of the terminal differentiation markers $\alpha$ - and $\beta$-MHC (Supplemental Figure 2). Therefore, we hypothesized that ERK1/2 hyperactivation affected cardiomyocyte cell cycling or proliferation. In $\beta$-MHC Q79R hearts, enhanced staining with the cell proliferation marker Ki-67 was apparent, particularly in the noncompacted ventricle (Figure 6A) and in the cells located at the boundaries of the muscular ventricular septum 
A

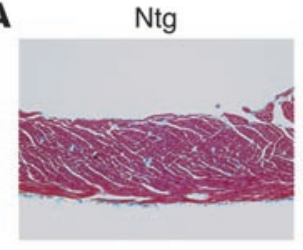

$\beta-\mathrm{MHC}$ Q79R

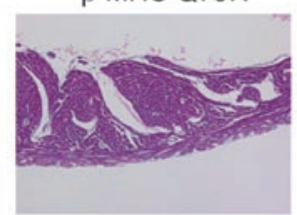

B

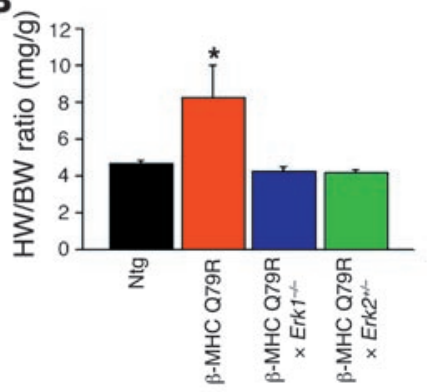

Erk1 $1^{-1-}$

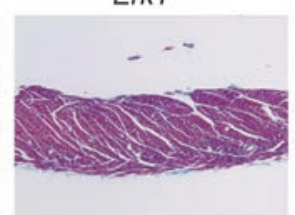

$\beta-M H C$ Q79R $\times E r k 1^{-1-}$
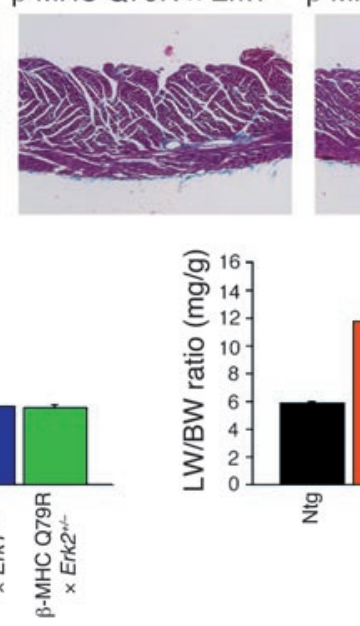

Erk2 $^{+/-}$

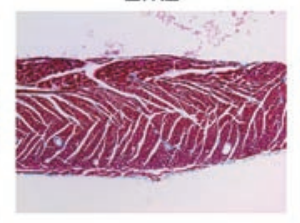

$\beta-\mathrm{MHC}$ Q79R $\times E r k 2^{+/}$

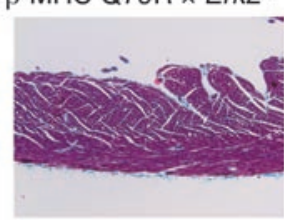

and atrioventricular cushion tissue (Figure 6B). However, normal levels of Ki-67 were restored in the $\beta$-MHC Q79R $\times$ Erk1 $^{-1-}$ and $\beta$-MHC Q79R $\times$ Erk2 $^{+/}$- hearts (Figure 6, A-C). These data suggest that enhanced cardiomyocyte proliferation or alterations in cell-cycling kinetics through ERK1/2 hyperactivation unfavorably impact normal ventricular maturation and that ablation of abnormal ERK1/2 activation can prevent the occurrence of Q79R-mediated cardiac malformations.

\section{Discussion}

Recent human genetic analyses have begun to define the involvement of Ras/MAPK pathway mutations in causing various congenital diseases, including the neurofibromatosis type 1 (NF1), cardio-facio-cutaneous, and Costello syndromes (34-37). Additionally, mutations in SHP2 can also cause LEOPARD syndrome (38) and, in at least some cases, in contrast to the Q79R mutation studied above, these mutations lead to lower than normal PTP activity $(39,40)$. The multiple effects of up- or downregulation of

\section{Figure 5}

Analyses of adult hearts. (A) Histology of 3-month-old hearts stained with Masson trichrome (right-ventricular free wall; original magnification, $\times 10)$. Rescued ventricular noncompaction observed in the embryonic stage persists postnatally in the $\beta-\mathrm{MHC}$ Q79R $\times \mathrm{Erk1}^{-1-}$ and $\beta-\mathrm{MHC}$ Q79R $\times \mathrm{Erk2}^{+/-}$hearts. All samples were taken from the identical location in the right ventricle, as denoted by the rectangle in Figure 2B. (B) HW/BW and LW/BW ratios in age- and sex-matched mice ( 3 months after birth; $n=8$ [4 male, 4 female]). ${ }^{*} P<0.05$.

SHP2 and the many similarities in the craniofacial, skeletal, and cardiovascular anomalies underscore the pleiotropic nature of Ras/MAPK activity in the different tissue types in which these human syndromes present.

Because the Q79R SHP2 mutation leads to a gain of function, we chose to use a transgenic approach in which gene dosage and protein expression could be varied between lines and expression controlled through the use of developmental stage-specific cardiac promoters. While this approach has a number of strengths, it does present some caveats, the most serious being the development of a phenotype merely through the expression of an abundance of protein due to transgenically driven overexpression. We attempted to control for this by matching transgenic expression levels in both the mutant Q79R and WT lines. The lack of a phenotype when the normal protein is overexpressed does confirm that the observed phenotype is specific to the Q79R allele, but we cannot rule out the possibility that if only half of the SHP2 protein in cardiomyocytes consisted of Q79R, no phenotype would present.

Ventricular trabeculation and compaction and closure of the ventricular septum are important morphogenic processes that are closely associated with cardiac growth regulation during mid- and late gestation $(41,42)$. Ventricular noncompaction is a unique type of cardiac disease generally found in infants and is thought to be caused by abnormal cardiomyocyte proliferation and/or maturation. Clinical manifestations are highly variable, ranging from no symptoms to heart failure, with potential complications that include arrhythmias and thromboembolic events. Strikingly, it is often associated with other cardiac malformations, such as VSDs and pulmonic stenosis (43). Various gene mutations can cause ventricular noncompaction. For instance, neuregulin signaling through the ErbB receptors can induce neonatal cardiomyocyte hypertrophy in culture through the ERK1/2 pathway,

Table 2

Reduction of ERK1/2 hyperphosphorylation restores cardiac function

\begin{tabular}{|c|c|c|c|c|c|c|}
\hline & Ntg & Erk1-/- & Erk2+/- & $\beta$-MHC Q79R & $\beta-\mathrm{MHC}$ Q79R $\times$ Erk1-/ & $\beta-\mathrm{MHC}$ Q79R $\times \mathrm{Erk2}^{+-}$ \\
\hline Heart rate (bpm) & $713 \pm 14$ & $703 \pm 13$ & $721 \pm 8$ & $698 \pm 8$ & $737 \pm 13$ & $731 \pm 19$ \\
\hline IVST (mm) & $0.74 \pm 0.01$ & $0.69 \pm 0.02$ & $0.64 \pm 0.02$ & $0.55 \pm 0.03^{\mathrm{A}, \mathrm{B}}$ & $0.64 \pm 0.03$ & $0.66 \pm 0.03$ \\
\hline LVPWT (mm) & $0.76 \pm 0.02$ & $0.72 \pm 0.02$ & $0.64 \pm 0.02$ & $0.59 \pm 0.03^{A, B}$ & $0.66 \pm 0.02$ & $0.64 \pm 0.03$ \\
\hline LVDd (mm) & $3.22 \pm 0.08$ & $3.56 \pm 0.14$ & $3.29 \pm 0.09$ & $3.62 \pm 0.12$ & $3.58 \pm 0.17$ & $3.47 \pm 0.16$ \\
\hline LVDs (mm) & $1.72 \pm 0.08$ & $2.00 \pm 0.20$ & $1.98 \pm 0.11$ & $2.82 \pm 0.14^{A, B, B, D, E}$ & $2.05 \pm 0.12$ & $2.06 \pm 0.17$ \\
\hline FS (\%) & $46.64 \pm 1.63$ & $44.64 \pm 3.37$ & $40.22 \pm 1.74$ & $22.22 \pm 1.84 \mathrm{~A}, \mathrm{~B}, \mathrm{C}, \mathrm{D}, \mathrm{E}$ & $43.00 \pm 0.90$ & $41.21 \pm 2.40$ \\
\hline Vcfc & $10.80 \pm 0.49$ & $9.69 \pm 0.74$ & $9.49 \pm 0.47$ & $4.41 \pm 0.83^{\mathrm{A}, \mathrm{B}, \mathrm{C}, \mathrm{D}, \mathrm{E}}$ & $9.48 \pm 0.16$ & $9.64 \pm 0.58$ \\
\hline
\end{tabular}

Age- and sex-matched mice (15 weeks after birth; $n=8$ [ 4 male, 4 female]) were assessed by M-mode echocardiography. Echocardiographic measurements and statistical analyses were performed as described in Table 1 and Methods, with the Ntg and $\beta$-MHC Q79R data taken from Table 1. AP $<0.05$

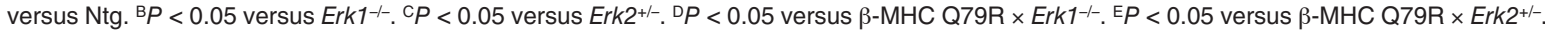



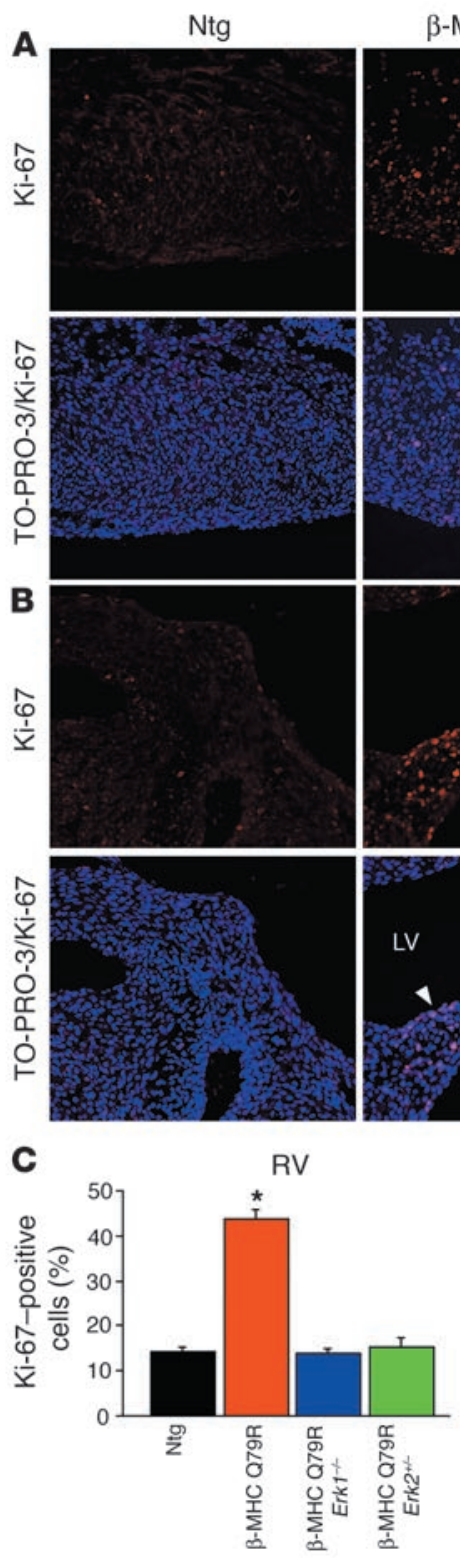

B-MHC Q79R
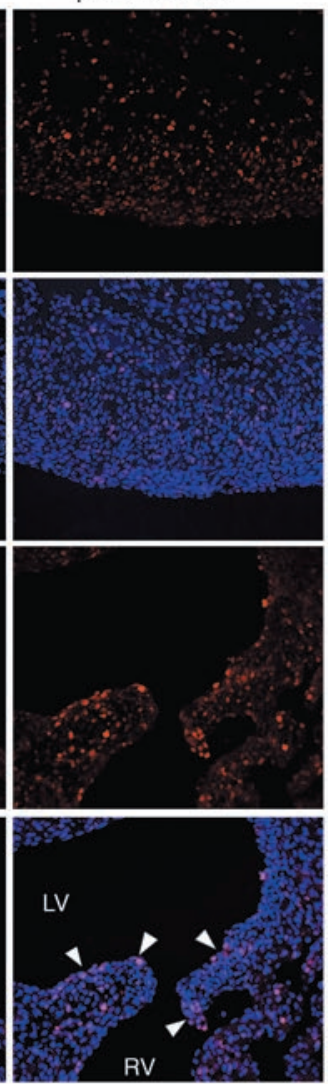

$\beta-M H C$ Q79R $\times E r k 1^{--}$
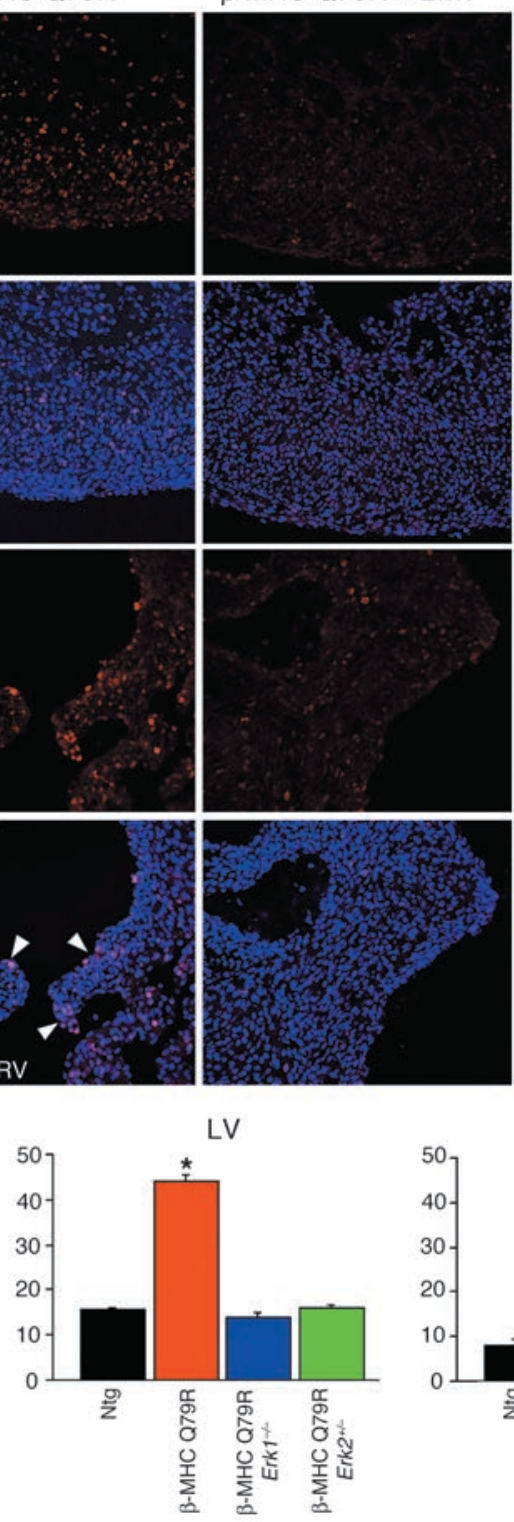

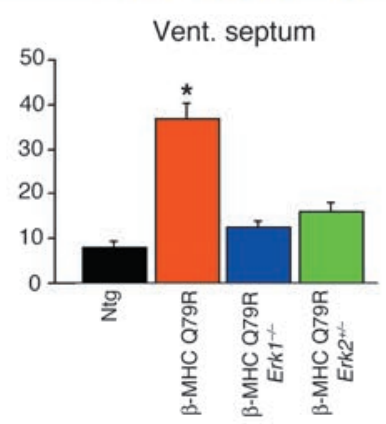

$\beta-\mathrm{MHC}$ Q79R $\times \mathrm{Erk2}^{+/-}$
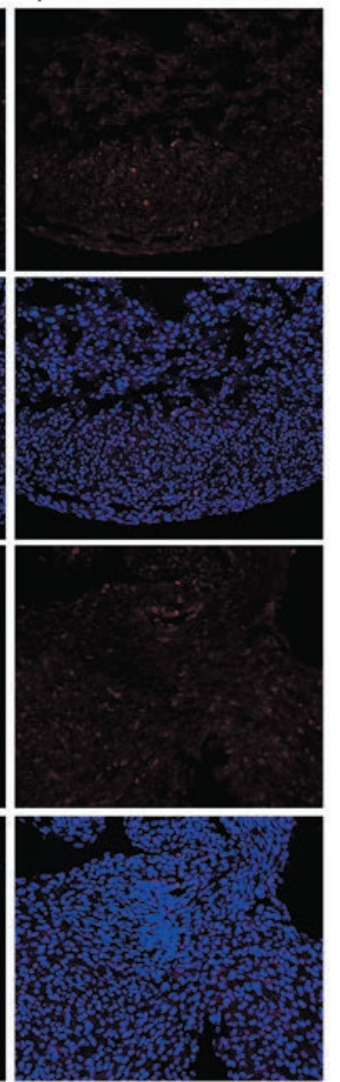

Vent. septum

\section{Figure 6}

Reduction of ERK1/2 hyperphosphorylation inhibits enhanced cardiomyocyte proliferation. (A and B) Ki-67 immunostaining (E16.5; original magnification, $\times 20)$. Ki-67-positive cardiomyocytes are abundant in the $\beta$-MHC Q79R hearts, particularly in the trabecular myocardium (A) and at the tip of the muscular ventricular septum (B, arrowheads). Normal cardiomyocyte proliferation is restored in the Erk1 $1^{-/}$and $\mathrm{Erk2}^{+/-}$backgrounds. (C) Quantitation of Ki-67-positive cells $(n=5)$. ${ }^{*} P<0.0001$. A total of 4,500 cells were counted in left, right, and septal areas of 5 hearts from each group. and both neuregulin- and ErbB4-deficient embryos lack mature trabecular myocardium $(44,45)$. Mouse embryos lacking the retinoic acid receptor gene Rxra show substantially reduced ERK1/2 activity, leading to a thin-walled noncompaction ventricle with a poorly formed interventricular septum (46). Foxp1-null mice also showed noncompaction, VSDs, and increased cardiomyocyte proliferation in the trabeculae (47). Our findings, in which enhanced ERK1/2 activation in the cardiomyocytes resulted in noncompaction, imply that the pathway may only operate within narrow limits for normal development to occur, although an alternative explanation for the apparent discrepancy may lie in the systemic expression of the null alleles versus the cardiomyocyte-specific activation of ERK1/ 2 in our model. In the $\beta$-MHC Q79R embryonic hearts, the number of cardiomyocytes in active phases of the cell cycle (i.e., Ki-67 positive) was increased as a result of ERK1/2 activation downstream of SHP2 hyperactivation. Normally, transient ERK1/2 activation is required for cardiac induction (31), and proliferative activity is lower in trabecular myocytes than in the compact wall (41). Our results show that enhanced cardiomyocyte proliferation through sustained ERK1/2 activation, particularly in the trabecular myocardium and in the regions surrounding the incipient VSD (Figure 6), inhibit ventricular compaction and closure of the ventricular septum.

Cardiomyocytes cease proliferation soon after birth, but neonatal cardiomyocytes retain the ability to respond to a variety of stimuli by hypertrophic growth (48). Activation of ERK1/2 signaling is often associated with cardiac hypertrophy in the adult heart (49), and we expected that ERK1/2 activation in the postnatal cardiomyocytes would result in HCM, which does present in a minority of NS patients. However, despite ERK1/2 activation, no hypertrophy was observed in either the $\alpha$ - or $\beta$-MHC Q79R hearts, confirming that ERK1/2 signaling mediated through SHP2 gain of function is not sufficient to trigger a cardiomyocyte-autonomous hypertrophic response. Consistent with these data, clinical studies have now confirmed a low incidence of HCM in NS patients carrying SHP2 mutations $(17,50)$ and in NF1 patients $(51)$. In contrast, 
there is a high incidence of HCM in LEOPARD syndrome patients carrying an SHP2 mutation located in the PTP domain $(38,52)$. The heterogeneous cardiac phenotypes may be explained by the distinct domains present in SHP2, such that the different mutations that occur will result in substantial differences in the degree of basal activity, susceptibility to activation, and possibly substrate specificity (53). Recently, mutations in other components of the Ras/ERK pathway have been linked to NS. For example, gain-offunction mutations in son of sevenless (SOS), a Ras-specific guanine nucleotide exchange factor, cause a distinctive form of NS (54, 55). A relatively higher incidence of HCM was noted in one of these studies (54), implying that mutations in genes other than PTPN11 underlie a majority of the hypertrophic phenotypes observed. Similar to the mouse model presented herein, some NS cases also show the presence of ventricular noncompaction or hypoplasia (56-58). Despite recent advances in imaging, ventricular noncompaction is still commonly misdiagnosed even if a skilled echocardiographer with previous experience with the condition is involved (59-61), and this may result in underestimation of the presence of ventricular noncompaction in NS patients.

Ras/MAPK signaling is activated in most human tumors, and clinical trials of Ras/MAPK inhibitors are underway (62). Possibly, therapeutic strategies might be devised that could mitigate some symptoms of the congenital syndromes as well. Ismat et al. recently reported that restoration of Ras/GTPase-activating protein (Ras/ GAP) activity through expression of the human NF1 GAP-related domain was able to normalize cardiac formation in Nf1-null mouse embryos (63). Our results consistently showed that downregulating the enhanced, cardiomyocyte-specific ERK1/2 signaling restored normal cardiac architecture and function, confirming the pathway's necessity in signaling the mutant SHP2's pathological effects on cardiovascular development. Strikingly, it appears that enhanced signaling through both ERK1 and ERK2 is necessary for the downstream effects of mutant SHP2 expression in the cardiomyocytes, as crossing to either the Erk1 ${ }^{-/-}$or Erk2 ${ }^{+/-}$lines was effective at mitigating cardiac pathology, implying that at least some substrate specificity for the 2 ERKs exists. The common and specific roles for each of the ERKs remain unclear (31), but our data are consistent with the hypothesis that specific downstream targets for each ERK exist in the cardiomyocytes. Although a deeper understanding of the biological functions of aberrant Ras/MAPK pathway signaling will be needed, our findings suggest that modulation of these signaling pathways could be effective and point to the potential development of therapeutic strategies in NS.

\section{Methods}

Production of Tg mice. Animal use was in accordance with Cincinnati Children's Hospital Medical Center institutional guidelines, with all experiments reviewed and approved by their Institutional Animal Care Committee. Mouse SHP2 cDNA was cloned using RT-PCR and multiple isolates sequenced (NCBI nucleotide sequence number NM_011202). Q79R SHP2 was made from the WT SHP2 cDNA using PCR-based mutagenesis. Subsequently, the WT and Q79R SHP2 were placed in the $\alpha$ - and $\beta$-MHC promoters and Tg mice generated. The PCR sequences employed for genotyping were: sense $5^{\prime}$-GGTCATGCGTGTTAGGAAC-3', antisense 5'-TTGTCTGGTCCACCAAGGAATAC-3'. All lines of Tg and gene-targeted mice were made in or backcrossed onto an FVBN background for at least 7 generations before being used in the crosses that were analyzed.

Histology and immunohistochemistry. All hearts used for histochemistry were fixed in the relaxed state by infusion of $4 \%$ formaldehyde in cardio- plegic buffer ( $50 \mathrm{mM} \mathrm{KCl}$ in $5 \%$ dextrose) from the right atrium while the heart was still beating. Hearts were then fixed with $4 \%$ paraformaldehyde in PBS overnight, embedded in paraffin, sectioned at a thickness of $5 \mu \mathrm{m}$, and stained with $\mathrm{H} \& \mathrm{E}$ and Masson trichrome. The sections used were analyzed according to strict diagnostic criteria: the presence of atrial septal defects/VSDs was determined only in sections in which the atrial septum, ventricular septum, and basal myocardium of both ventricles were present in a single plane. Sections used for quantifying ventricular noncompaction were taken in all cases exactly in the middle part of the heart where the right- and left-ventricular cavity areas were largest and all leaflets of both mitral and tricuspid valves were seen in the same plane. These sectioning criteria avoided any artifacts that might result from an oblique cutting angle. The diagnostic criteria correspond to the "apical chamber view" used during clinical ultrasound. All hearts were entirely and consecutively sectioned (5- $\mu \mathrm{m}$ thickness): at least 4 hearts for each line were sectioned in this manner and 3 sections analyzed for each heart. Using those sections, the entire left- and right-ventricular free wall areas were determined using MetaMorph (version 6.3; Molecular Devices). A rectangle encompassing the entire free wall was drawn and the number of pixels shown in the myocardium (orange; Figure 4C) determined. The compaction index was then defined as the area of the orange region divided by the area of the region of interest. Using this method, we have obtained highly reproducible results in comparisons of hearts from the same group. A heart was defined as being noncompacted if the value of its index fell 2 SDs below the index obtained from at least $5 \mathrm{Ntg}$ hearts of the same age.

For immunohistochemistry, embryonic hearts were fixed overnight in $4 \%$ paraformaldehyde in PBS at $0^{\circ} \mathrm{C}$ and embedded in OCT as described previously (64), and $5-\mu \mathrm{m}$ sections were cut from the frozen block. Primary antibodies used were: (a) goat anti-SH-PTP2 (E-20; 1:100 dilution; Santa Cruz Biotechnology Inc.) to identify SHP2; (b) rabbit anti-cleaved caspase-3 (1:100 dilution; Cell Signaling Technology); (c) rabbit anti-Ki-67 (1:1,000 dilution; Abcam) to examine cardiomyocyte proliferation. Donkey anti-goat Alexa Fluor 488 or goat anti-rabbit Alexa Fluor 568 (1:100 dilution; Molecular Probes; Invitrogen) was used as a secondary antibody. Nuclei were stained with TO-PRO-3 (1:200 dilution; Molecular Probes; Invitrogen) for 20 minutes.

Protein analyses. Protein extracts were prepared from myocardial homogenates derived from between 5 (at E16.5) and 20 (at E9.5) embryonic hearts. Ventricular samples were homogenized on ice in RIPA buffer with $1 \%$ phosphatase inhibitor cocktail (Sigma-Aldrich). The homogenates were centrifuged $\left(8,000 \mathrm{~g}\right.$ for 10 minutes at $\left.4^{\circ} \mathrm{C}\right)$, and the supernatant was mixed with SDS-PAGE loading buffer and then boiled and centrifuged. Antibodies used were as follows: SH-PTP2 (C-18), MYPT1 (1:500 dilution; Santa Cruz Biotechnology Inc.), p44/42 MAPK, phospho-p44/42 MAPK, p38, phospho-p38, JNK1/2, phospho-JNK1/2, ERK5, phospho-ERK5, Akt, Ser473-phospho-Akt, STAT1, phospho-STAT1, STAT3, phospho-STAT3, phospho-STAT5, caspase-3 (1:1,000 dilution; Cell Signaling Technology), phospho-MYPT1 and STAT5B (1:3,000 dilution; Upstate; Millipore), and GAPDH (1:300 dilution; Chemicon; Millipore). Primary antibody binding was visualized by HRP-conjugated secondary antibodies and enhanced chemiluminescence (Amersham; GE Healthcare). Nonphosphorylated and phosphorylated species were visualized and quantitated in a Storm PhosphorImager (Molecular Devices; GE Healthcare Life Sciences).

$R T-P C R$. Three micrograms of RNA was subjected to an RT reaction, and the resulting cDNA was obtained using SuperScript III (Invitrogen) as described previously (64). PCR analysis of differentiation markers was performed using their specific primers. PCR products were analyzed after serial cycles in the linear range of amplification. The products were loaded on $2 \%$ agarose gel, and the image was obtained using a Typhoon 9400 scan- 
ner (Amersham; GE Healthcare). The relative expression was normalized to the reference gene ribosomal protein L7. The primer sets were as follows: (a) $\alpha$-MHC forward primer (5'-ACCTACCAGACAGAGGAAGA-3'), reverse primer ( $5^{\prime}$-ATTGTGTATTGGCCACAGCG-3'); (b) $\beta$-MHC forward primer (5'-ACCTACCAGACAGAGGAAGA), reverse primer (5'-TTGCAAAGAGTCCAGGTCTGAG-3'); (c) L7 forward primer (5'-GAAGCTCATCTATGAGAAGGC-3'), reverse primer (5'-AAGACGAAGGAGCTGCAGAAC-3').

Echocardiographic analysis. Echocardiographic measurements were performed in conscious animals with a Hewlett Packard Sonos 5500 instrument and the hearts visualized with a $15-\mathrm{MHz}$ transducer. Measurements were averaged from at least 3 separate cardiac cycles.

Statistics. All data are expressed as mean \pm SEM. Statistical significance was determined using the 2-tailed Student's $t$ test or ANOVA, as appropriate. The significance of individual differences was evaluated using the
Scheffé test if ANOVA was significant. A $P$ value of less than 0.05 was considered significant.

\section{Acknowledgments}

This work was supported by NIH research grants R01 HL69799, HL60546, HL52318, and HL56370 (to J. Robbins).

Received for publication October 25, 2006, and accepted in revised form May 8, 2007.

Address correspondence to: Jeffrey Robbins, Cincinnati Children's Hospital Medical Center, MLC 7020, 3333 Burnet Avenue, Cincinnati, Ohio 45229-3039, USA. Phone: (513) 636-8098; Fax: (513) 636-5958; E-mail: jeff.robbins@cchmc.org.
1. Allanson, J.E. 1987. Noonan syndrome. J. Med. Genet. 24:9-13.

2. Noonan, J.A. 1994. Noonan syndrome. An update and review for the primary pediatrician. Clin. Pediatr. (Phila.). 33:548-555.

3. Sharland, M., Burch, M., McKenna, W.M., and Paton, M.A. 1992. A clinical study of Noonan syndrome. Arch. Dis. Child. 67:178-183.

4. Bader-Meunier, B., et al. 1997. Occurrence of myeloproliferative disorder in patients with Noonan syndrome. J. Pediatr. 130:885-889.

5. Choong, K., et al. 1999. Juvenile myelomonocytic leukemia and Noonan syndrome. J. Pediatr. Hematol. Oncol. 21:523-527.

6. Kramer, H.H., Majewski, F., Trampisch, H.J., Rammos, S., and Bourgeois, M. 1987. Malformation patterns in children with congenital heart disease. Am. J. Dis. Child. 141:789-795.

7. Marino, B., Digilio, M.C., Toscano, A., Giannotti, A., and Dallapiccola, B. 1999. Congenital heart diseases in children with Noonan syndrome: an expanded cardiac spectrum with high prevalence of atrioventricular canal. J. Pediatr. 135:703-706.

8. Tartaglia, M., et al. 2001. Mutations in PTPN11, encoding the protein tyrosine phosphatase SHP-2, cause Noonan syndrome. Nat. Genet. 29:465-468.

9. Tang, T.L., Freeman, R.M., Jr., O’Reilly, A.M., Neel, B.G., and Sokol, S.Y. 1995. The SH2-containing protein-tyrosine phosphatase SH-PTP2 is required upstream of MAP kinase for early Xenopus development. Cell. 80:473-483.

10. Yang, W., et al. 2006. An Shp2/SFK/Ras/Erk signaling pathway controls trophoblast stem cell survival. Dev. Cell. 10:317-327.

11. Saxton, T.M., et al. 2000. The SH2 tyrosine phosphatase shp2 is required for mammalian limb development. Nat. Genet. 24:420-423.

12. Chen, B., et al. 2000. Mice mutant for Egfr and Shp2 have defective cardiac semilunar valvulogenesis. Nat. Genet. 24:296-299.

13. Chan, R.J., Johnson, S.A., Li, Y., Yoder, M.C., and Feng, G.S. 2003. A definitive role of Shp-2 tyrosine phosphatase in mediating embryonic stem cell differentiation and hematopoiesis. Blood. 102:2074-2080.

14. Qu, C.K., and Feng, G.S. 1998. Shp-2 has a positive regulatory role in ES cell differentiation and proliferation. Oncogene. 17:433-439.

15. Neel, B.G., Gu, H., and Pao, L. 2003. The 'Shp'ing news: $\mathrm{SH} 2$ domain-containing tyrosine phosphatases in cell signaling. Trends Biochem. Sci. 28:284-293.

16. O'Reilly, A.M., Pluskey, S., Shoelson, S.E., and Neel, B.G. 2000. Activated mutants of SHP-2 preferentially induce elongation of Xenopus animal caps. Mol. Cell. Biol. 20:299-311.

17. Tartaglia, M., et al. 2002. PTPN11 mutations in Noonan syndrome: molecular spectrum, genotypephenotype correlation, and phenotypic heterogeneity. Am.J. Hum. Genet. 70:1555-1563.
18. Araki, T., et al. 2004. Mouse model of Noonan syndrome reveals cell type- and gene dosage-dependent effects of Ptpn11 mutation. Nat. Med. 10:849-857.

19. Krenz, M., Yutzey, K.E., and Robbins, J. 2005. Noonan syndrome mutation Q79R in Shp2 increases proliferation of valve primordia mesenchymal cells via extracellular signal-regulated kinase $1 / 2$ signaling. Circ. Res. 97:813-820.

20. Uhlen, P., et al. 2006. Gain-of-function/Noonan syndrome SHP-2/Ptpn11 mutants enhance calcium oscillations and impair NFAT signaling. Proc. Natl. Acad. Sci. U. S. A. 103:2160-2165.

21. Feng, G.S. 1999. Shp-2 tyrosine phosphatase: signaling one cell or many. Exp. Cell Res. 253:47-54.

22. Nakaoka, Y., et al. 2003. Activation of gp130 transduces hypertrophic signal through interaction of scaffolding/docking protein Gab1 with tyrosine phosphatase SHP2 in cardiomyocytes. Circ. Res. 93:221-229.

23. Ruhul Amin, A.R., et al. 2003. SH2 domain containing protein tyrosine phosphatase 2 regulates concanavalin A-dependent secretion and activation of matrix metalloproteinase 2 via the extracellular signal-regulated kinase and p38 pathways. Cancer Res. 63:6334-6339.

24. Shi, Z.Q., Lu, W., and Feng, G.S. 1998. The Shp-2 tyrosine phosphatase has opposite effects in mediating the activation of extracellular signal-regulated and c-Jun NH2-terminal mitogen-activated protein kinases. J. Biol. Chem. 273:4904-4908.

25. Ng, W.A., Grupp, I.L., Subramaniam, A., and Robbins, J. 1991. Cardiac myosin heavy chain mRNA expression and myocardial function in the mouse heart. Circ. Res. 68:1742-1750.

26. Ivins Zito, C., Kontaridis, M.I., Fornaro, M., Feng, G.S., and Bennett, A.M. 2004. SHP-2 regulates the phosphatidylinositide 3'-kinase/Akt pathway and suppresses caspase 3-mediated apoptosis. J. Cell. Physiol. 199:227-236.

27. You, M., Yu, D.H., and Feng, G.S. 1999. Shp-2 tyrosine phosphatase functions as a negative regulator of the interferon-stimulated Jak/STAT pathway. Mol. Cell. Biol. 19:2416-2424.

28. Schoenwaelder, S.M., et al. 2000. The protein tyrosine phosphatase Shp-2 regulates RhoA activity. Curr. Biol. 10:1523-1526.

29. Kontaridis, M.I., et al. 2004. SHP-2 positively regulates myogenesis by coupling to the Rho GTPase signaling pathway. Mol. Cell. Biol. 24:5340-5352.

30. Saba-El-Leil, M.K., et al. 2003. An essential function of the mitogen-activated protein kinase Erk2 in mouse trophoblast development. EMBO Rep. 4:964-968.

31. Corson, L.B., Yamanaka, Y., Lai, K.M., and Rossant, J. 2003. Spatial and temporal patterns of ERK signaling during mouse embryogenesis. Development. 130:4527-4537.

32. Ebisuya, M., Kondoh, K., and Nishida, E. 2005. The duration, magnitude and compartmentalization of
ERK MAP kinase activity: mechanisms for providing signaling specificity. J. Cell Sci. 118:2997-3002.

33. Pages, G., et al. 1999. Defective thymocyte maturation in $\mathrm{P} 44 \mathrm{MAP}$ kinase (Erk 1) knockout mice. Science. 286:1374-1377.

34. Aoki, Y., et al. 2005. Germline mutations in HRAS proto-oncogene cause Costello syndrome. Nat. Genet. 37:1038-1040.

35. Cichowski, K., and Jacks, T. 2001. NF1 tumor suppressor gene function: narrowing the GAP. Cell. 104:593-604

36. Niihori, T., et al. 2006. Germline KRAS and BRAF mutations in cardio-facio-cutaneous syndrome. Nat. Genet. 38:294-296.

37. Rodriguez-Viciana, P., et al. 2006. Germline mutations in genes within the MAPK pathway cause cardio-facio-cutaneous syndrome. Science. 311:1287-1290.

38. Digilio, M.C., et al. 2002. Grouping of multiple-lentigines/LEOPARD and Noonan syndromes on the PTPN11 gene. Am.J. Hum. Genet. 71:389-394.

39. Kontaridis, M.I., Swanson, K.D., David, F.S., Barford, D., and Neel, B.G. 2006. PTPN11 (Shp2) mutations in LEOPARD syndrome have dominant negative, not activating, effects. J. Biol. Chem. 281:6785-6792.

40. Tartaglia, M., et al. 2006. Diversity and functional consequences of germline and somatic PTPN11 mutations in human disease. Am. J. Hum. Genet. 78:279-290.

41. Sedmera, D., Pexieder, T., Vuillemin, M., Thompson, R.P., and Anderson, R.H. 2000. Developmental patterning of the myocardium. Anat. Rec. 258:319-337.

42. Webb, S., Brown, N.A., and Anderson, R.H. 1998. Formation of the atrioventricular septal structures in the normal mouse. Circ. Res. 82:645-656.

43. Weiford, B.C., Subbarao, V.D., and Mulhern, K.M. 2004. Noncompaction of the ventricular myocardium. Circulation. 109:2965-2971.

44. Gassmann, M., et al. 1995. Aberrant neural and cardiac development in mice lacking the ErbB4 neuregulin receptor. Nature. 378:390-394.

45. Kramer, R., et al. 1996. Neuregulins with an Ig-like domain are essential for mouse myocardial and neuronal development. Proc. Natl. Acad. Sci. U. S. A. 93:4833-4838.

46. Sucov, H.M., et al. 1994. RXR alpha mutant mice establish a genetic basis for vitamin A signaling in heart morphogenesis. Genes Dev. 8:1007-1018.

47. Wang, B., et al. 2004. Foxp1 regulates cardiac outflow tract, endocardial cushion morphogenesis and myocyte proliferation and maturation. Development. 131:4477-4487.

48. MacLellan, W.R., and Schneider, M.D. 2000. Genetic dissection of cardiac growth control pathways. Annu. Rev. Physiol. 62:289-319.

49. Bueno, O.F., et al. 2000. The MEK1-ERK1/2 signaling pathway promotes compensated cardiac hypertrophy in transgenic mice. EMBOJ. 19:6341-6350. 
50. Jongmans, M., et al. 2005. Genotypic and phenotypic characterization of Noonan syndrome: new data and review of the literature. Am. J. Med. Genet. A. 134:165-170.

51. Lin, A.E., et al. 2000. Cardiovascular malformations and other cardiovascular abnormalities in neurofibromatosis 1. Am. J. Med. Genet. 95:108-117.

52. Sarkozy, A., et al. 2003. Correlation between PTPN11 gene mutations and congenital heart defects in Noonan and LEOPARD syndromes. J. Med. Genet. 40:704-708.

53. Keilhack, H., David, F.S., McGregor, M., Cantley, L.C., and Neel, B.G. 2005. Diverse biochemical properties of Shp2 mutants. Implications for disease phenotypes. J. Biol. Chem. 280:30984-30993.

54. Roberts, A.E., et al. 2007. Germline gain-of-function mutations in SOS1 cause Noonan syndrome. Nat. Genet. 39:70-74.

55. Tartaglia, M., et al. 2007. Gain-of-function SOS1 mutations cause a distinctive form of Noonan syndrome. Nat. Genet. 39:75-79.

56. Amann, G., and Sherman, F.S. 1992. Myocardial dysgenesis with persistent sinusoids in a neonate with Noonan's phenotype. Pediatr. Pathol. 12:83-92.

57. Antonelli, D., Antonelli, J., and Rosenfeld, T. 1990. Noonan's syndrome associated with hypoplastic left heart. Cardiology. 77:62-65.

58. Wilmshurst, P., and Da Costa, P. 1995. Probable right ventricular dysplasia and patent foramen ovale presenting with cyanosis and clubbing in a patient with characteristics of Noonan syndrome. Br. Heart J. 74:471-475.

59. Bozic, I., Fabijanic, D., Carevic, V., and Polic, S. 2006. Echocardiography in the diagnosis and management of isolated left ventricular noncompaction: case reports and review of the literature. J. Clin. Ultrasound. 34:416-421.

60. McCrohon, J.A., Richmond, D.R., Pennell, D.J., and
Mohiaddin, R.H. 2002. Images in cardiovascular medicine. Isolated noncompaction of the myocardium: a rarity or missed diagnosis? Circulation. 106:e22-e23.

61. van der Loo, B., and Jenni, R. 2003. Isolated noncompaction of the myocardium. Circulation. 107:e50; author reply e50.

62. Sebolt-Leopold, J.S., and Herrera, R. 2004. Targeting the mitogen-activated protein kinase cascade to treat cancer. Nat. Rev. Cancer. 4:937-947.

63. Ismat, F.A., Xu, J., Lu, M.M., and Epstein, J.A. 2006. The neurofibromin GAP-related domain rescues endothelial but not neural crest development in $N f 1^{-/-}$mice. J. Clin. Invest. 116:2378-2384. doi:10.1172/JCI28341.

64. Nakamura, T., Colbert, M.C., and Robbins, J. 2006. Neural crest cells retain multipotential characteristics in the developing valves and label the cardiac conduction system. Circ. Res. 98:1547-1554. 